\title{
Modeling of GPR data in a stack of VTI-layers with an analytical code
}

\author{
J. Hunziker, J. Thorbecke and E. Slob \\ Delft University of Technology \\ Stevinweg 1, 2628CN, Delft, The Netherlands \\ j.w.hunziker@tudelft.nl, j.w.thorbecke@tudelft.nl, e.c.slob@tudelft.nl
}

\begin{abstract}
We developed an analytical code to model electromagnetic fields in 3D in a stack of vertical transverse isotropic layers. This code is suitable to model low-frequency as well as high-frequency responses and it is expected to become available as an open source package in 2014. We give an overview of the code and use it to investigate the sensitivity of different Ground Penetrating Radar antenna configurations to the horizontal and vertical electric permittivity. This investigation shows that a configuration with the source and the receiver antennas horizontal and orthogonal to the survey line is the most suitable for estimating the horizontal and vertical electric permittivity if data are only available for small offsets.
\end{abstract}

Index Terms-Vertical Transverse Isotropy (VTI), Ground Penetrating Radar (GPR), forward modeling.

\section{INTRODUCTION}

Over the last years, we have developed the forward modeling code EMmod to model electromagnetic fields in 3D in a stack of vertical transverse isotropic (VTI) layers. This code, written in $\mathrm{C}$ and Fortran, is supposed to become available as an open-source program somewhen in 2014 [1]. It was developed to model electromagnetic low-frequency data, like for example Controlled-Source Electromagnetics (CSEM) used by the hydrocarbon industry (for an overview see for example [2], [3]). At the typical frequencies of CSEM $(0.1 \mathrm{~Hz}$ to $10 \mathrm{~Hz}$ ) the electromagnetic field behaves as a diffusive field, which is quite different from the wave-behavior which can be observed at high frequencies. Nevertheless, the code is also able to model high-frequency data, like for example Ground Penetrating Radar (GPR) data. In this abstract, we give a short overview over the code for modeling GPR data. Further, we use the VTI capabilities of the code to investigate the sensitivity of the antenna orientations to vertical and horizontal electric permittivity.

\section{OVERVIEW OF THE CODE}

An outline of the code is sketched in Figure 1. Roughly, it can be said, that the algorithm consists of three major parts:

1) Everything before the Hankel-Transform loop

2) Everything in the Hankel-Transform loop

3) Everything after the Hankel-Transform loop

During the first major part (everything before the HankelTransform loop) all the parameters are read from the input file and other preparatory work is done. Then the electromagnetic field is computed in the frequency-wavenumber domain for the selected component, i.e., antenna orientation. The computation is done only for a line of wavenumbers. In the HankelTransform loop (the second part of the code), the Hankel transformation is carried out to bring the data from the 1D wavenumber domain to the 1D space domain. The integral in the Hankel transformation is computed using the 61-point Gauss-Kronrod rule from the slatec library [4]. In the final step of the Hankel-Transform loop it is assessed if enough points in the space domain have been computed to build up the 2D grid in the frequency-space domain from the transformed line of data. This assessment is done by interpolating the electromagnetic field at one point using the two neighboring points. If the interpolated point differs from the integrated electromagnetic field at that location by less than a predefined value, no additional points are required at that location. If the difference is larger, two more points are added. The Hankel transformation of the electromagnetic field at the new locations will be computed in the next iteration of the HankelTransform loop. The Hankel-Transform loop ends if all points of the line are within the required precision or if the Hankel transformation has been evaluated for more than a specified amount of points. In the third major part (everything after the Hankel-Transform loop) the electromagnetic field for one quadrant of the grid in the space domain is computed using the line data in the space domain and the corresponding cosine or sine factors. Next, the electromagnetic field for the complete grid is built up by copying and pasting of the quadrant already computed and by taking into account the symmetry of the field. Finally, the electromagnetic field in the frequency-space domain is written to the output file on disk and the program stops.

Because the code was written for monochromatic frequencydomain CSEM, the output is the electromagnetic field for one frequency in the frequency-space domain. GPR-data can be obtained by computing a set of frequencies (in our case 2048 frequencies) by calling EMmod in a loop and performing a Fourier transformation over these frequencies to obtain the electromagnetic field in the time-space domain.

\section{USING EMMOD FOR GPR}

Several studies have shown, that media investigated by GPR-surveys may exhibit some form of anisotropy like for example for glaciers [5] and [6], for wood [7] or for sandstone [8]. Therefore, it is important to take anisotropy into account in 


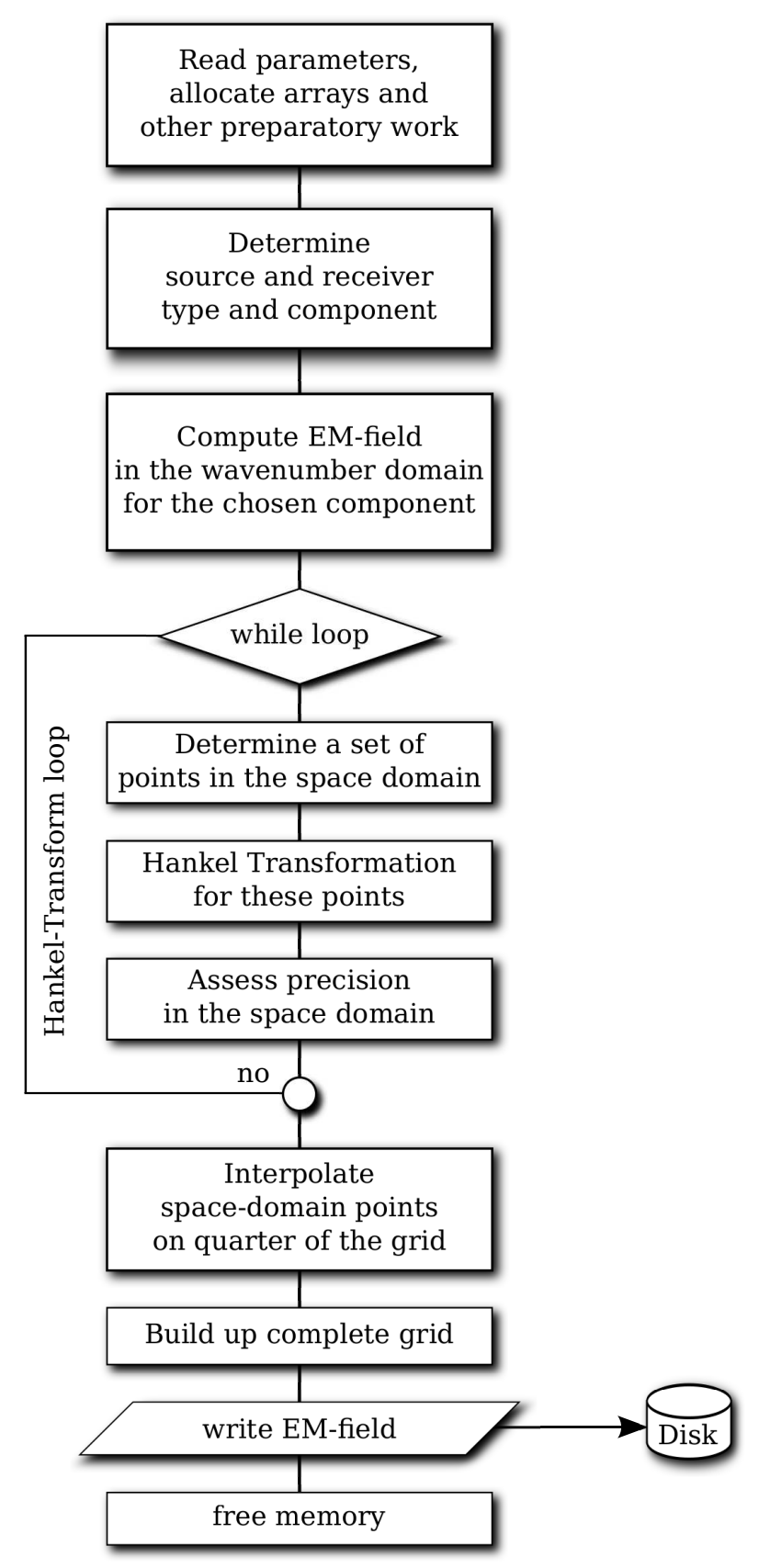

Fig. 1. Structure of EMmod program.

modeling (e.g., [9]) or inversion (e.g., [10]). Our code is able to model a VTI medium, which is a specific case of anisotropy featuring different material parameters in the horizontal and the vertical direction. In our code, a material is described by three parameters, which are electric conductivity, electric permittivity and magnetic permeability. All three parameters have a horizontal and a vertical component. Thus, a medium is effectively described by six parameters. This allows to simulate GPR data, for which the electric permittivity is the most important material parameter assuming the conductivity to be small.
The computation time depends mainly on the desired amount of gridpoints in the frequency-space domain and the desired precision of the Hankel transformation. The code requires only a few seconds to compute the electromagnetic field for one frequency, which is already a CSEM dataset. For GPR, many frequency slices of data need to be computed, which increases the computation time tremendously. In this abstract, we computed 2048 frequencies for each example. Computation time can be decreased by either parallelizing the code over frequencies or by reducing the amount of frequencies to be computed. The latter could be done, firstly, by limiting the band of computed frequencies and, secondly, by computing a random set of frequencies followed by an interpolation on a regular frequency grid.

When the source and the receiver are on the same depth level, the wavenumber spectrum of the direct field extends to infinity. This is a problem, because a numerical integration scheme can not integrate over an unlimited range of wavenumbers. The problem can be reduced by computing the direct field directly in the frequency-space domain and only the reflected field in the frequency-wavenumber domain. However, if the source and the receiver are located at the same depth and at the same time also close to an interface, not only the direct field is of infinite bandwidth, but also the specular reflection of that interface. In that situation our code requires to introduce a small vertical distance between the source and the receiver. The length of the integral in the wavenumber domain is an input parameter of the code. If the length of the integral is chosen large, smaller vertical distances between the source and the receiver are possible, but this also increases the computation time.

$$
\begin{aligned}
& \text { air: } \varepsilon_{\mathrm{r}}=1, \sigma=0 \mathrm{~S} / \mathrm{m} \\
& 0 \mathrm{~m} \frac{\text { sediment } 1: \varepsilon_{\mathrm{r}}=9, \sigma=0.005 \mathrm{~S} / \mathrm{m}}{\text { source }} \\
& \mathrm{h} \mathrm{m}-\underset{\text { sediment } 1: \varepsilon_{\mathrm{r}}=9, \sigma=0.005 \mathrm{~S} / \mathrm{m}}{-}-\text { receivers } \\
& 1 \mathrm{~m} \\
& \text { sediment 2: } \varepsilon_{\mathrm{r}}=14, \sigma=0.005 \mathrm{~S} / \mathrm{m}
\end{aligned}
$$

Fig. 2. Setup used for the modeling of the data. While the solid lines indicate interfaces with changes of relative electric permittivity $\varepsilon_{r}$ and electric conductivity $\sigma$ as specified in the figure, the dashed line indicates the receiver level, where no changes of material parameters occur. The source is at the surface at a depth of $0 \mathrm{~m}$.

To demonstrate this aspect, we modeled data for a simple model of one layer sandwiched by two homogeneous halfspaces with the source-receiver geometry and material parameters (relative electric permittivity $\varepsilon_{r}$ and electric conductivity $\sigma$ ) shown in Figure 2 . The magnetic permeability is the value of free space everywhere and the vertical and horizontal parameters for the electric permittivity and electric conductivity are equal. The center frequency of the source signal is $250 \mathrm{Mhz}$. Modeled data for receiver depths $h$ of 0.5 $\mathrm{m}, 0.2 \mathrm{~m}$ and $0.1 \mathrm{~m}$ (corresponding to $5 / 4,1 / 2$ and $1 / 4$ of the 
dominant wavelength) are shown in Figure 3. The different events are labeled and described in the figure caption. Because the data in Figure 3 are modeled with a constant wavenumber spectrum, artifacts are expected to appear with decreasing receiver depth. Artifacts are visible around zero offsets and at all times on all three plots. While they are barely visible for a receiver depth of $0.5 \mathrm{~m}$, they grow in amplitude for the receiver depths of $0.2 \mathrm{~m}$ and $0.1 \mathrm{~m}$. The artifacts would further grow in amplitude and spread to larger offsets if the receiver depth were reduced further. To ensure that these artifacts remain at low amplitude, either the wavenumber spectrum has to be increased, which results in larger computation time, or a relatively large receiver depth has to be chosen. We decided to apply the latter and use, for the remainder of this study, a receiver depth of $0.5 \mathrm{~m}$. This should not alter our conclusions about VTI sensitivity presented in the following section while reducing the computational time. Note, that the weak event which is dipping towards zero-offset and which is visible for all three cases after $70 \mathrm{~ns}$ is a numerical artifact unrelated to the vertical source-receiver distance.

\section{VTI-SENSITIVITY}

In order to invert GPR-data for the subsurface distribution of horizontal and vertical electric permittivity, a fast forwardmodeling code like EMmod is one key ingredient. But one also needs to know which antenna setup is most sensitive to which parameter in order to make the right acquisition choice. This is investigated with the following numerical experiment: For different antenna configurations the sensitivity $S$, which is the numerical derivative with respect to electric permittivity, is computed as follows:

$$
S=\frac{\partial E}{\partial \varepsilon_{r}^{H, V}}=\frac{E\left(\varepsilon_{r}^{H, V}+\Delta\right)-E\left(\varepsilon_{r}^{H, V}-\Delta\right)}{2 \Delta},
$$

where $E$ is the electric field in the space-time domain, $\varepsilon_{r}^{H, V}$ is the subsurface distribution of the relative horizontal (superscript $H$ ) or vertical (superscript $V$ ) electric permittivity and $\Delta$ is a small value, in our case 0.1 . The sensitivity with respect to the horizontal and vertical relative electric permittivity $\varepsilon_{r}^{H, V}$ of the first layer is shown in Figures $4 a$ and $4 c$ for the situation where the source- and the receiver-antennas are parallel to the survey line (xx-configuration). This antenna configuration has the largest sensitivity for the horizontal permittivity at short offsets $(<0.3 \mathrm{~m})$ and for the vertical permittivity at intermediate offsets $(>0.3 \mathrm{~m}$ and $<1 \mathrm{~m})$.

However, on these figures only the sensitivity due to the direct wave can be seen. By subtracting the sensitivity of a two-halfspace model, i.e. the model shown in Figure 2 without the interface at $1 \mathrm{~m}$ depth, from the data shown in Figures $4 \mathrm{a}$ and $4 c$, we obtain the sensitivity of the reflected signal. This is plotted for the horizontal and vertical permittivity of the first layer in Figures $4 \mathrm{~b}$ and $4 \mathrm{~d}$, respectively. Comparing Figure $4 \mathrm{a}$ with $4 \mathrm{~b}$ and $4 \mathrm{c}$ with $4 \mathrm{~d}$, we can see that the sensitivity of the direct field is at least one order of magnitude larger than the sensitivity of the reflected field, but, considering the
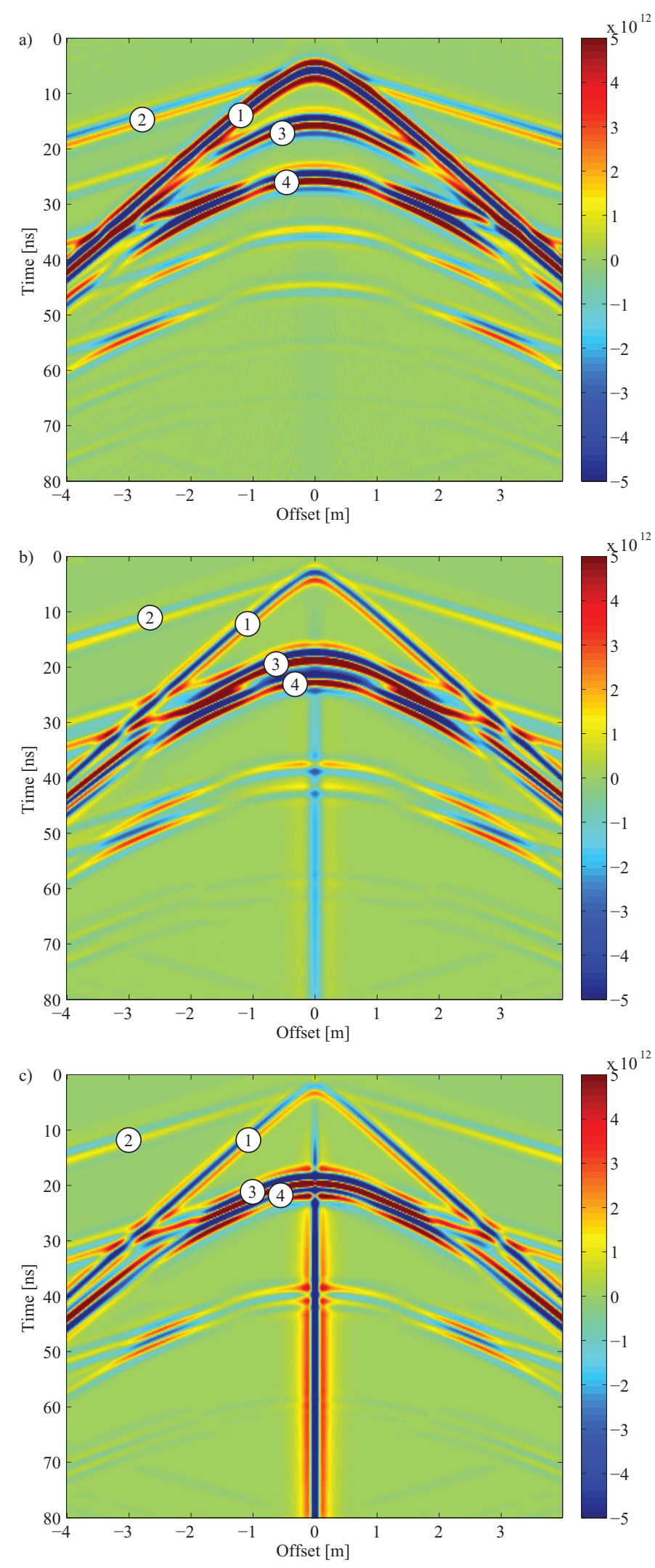

Fig. 3. Data for the model shown in Figure 2 for a receiver depth of a) 0.5 $\mathrm{m}$, b) $0.2 \mathrm{~m}$ and c) $0.1 \mathrm{~m}$ below the surface. The labels indicate different events: (1) is the direct wave, (2) is the surface refraction, (3) is the reflection of the subsurface interface and (4) is the first surface multiple of the subsurface reflection. 

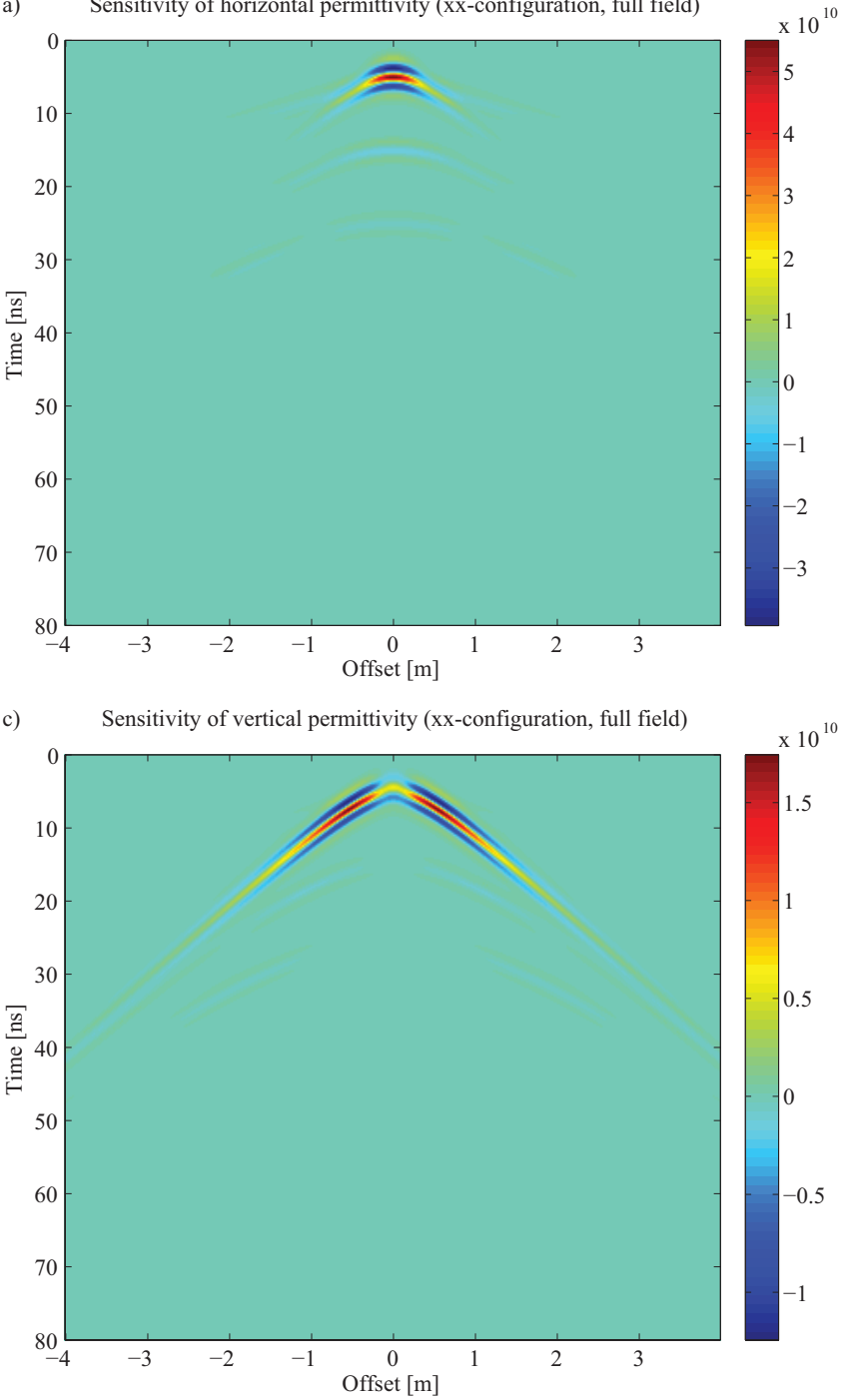

b) Sensitivity of horizontal permittivity (xx-configuration, reflections only)
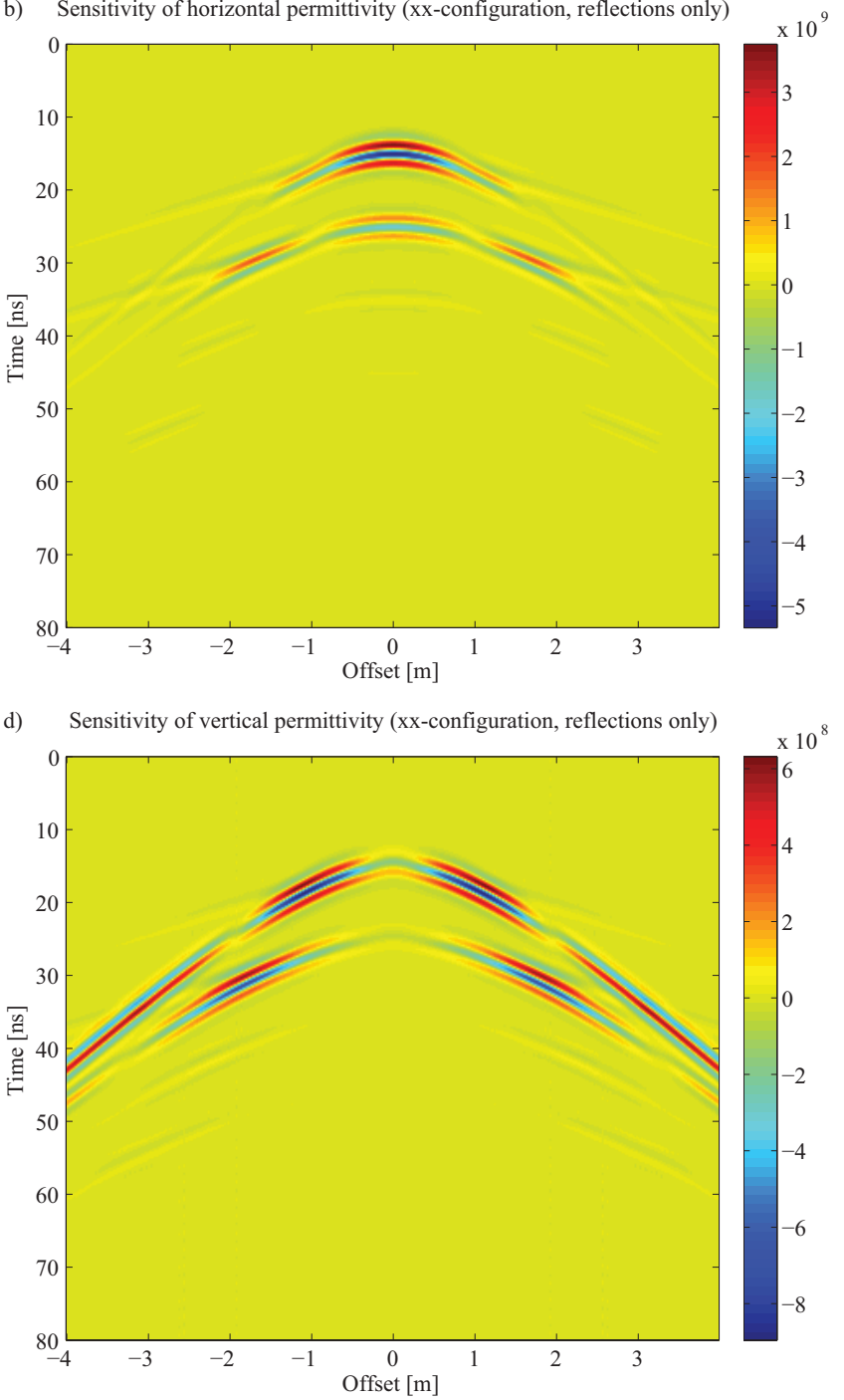

Fig. 4. Sensitivity to the electric permittivity of the first layer with the source- and the receiver-antennas oriented parallel to the survey line (xx-configuration): a) Sensitivity to the horizontal permittivity, b) sensitivity to the horizontal permittivity featuring only the reflections, c) sensitivity to the vertical permittivity and d) sensitivity to the vertical permittivity featuring only the reflections.

reflections, the area of increased sensitivity spreads to larger offsets.

The same computation of the sensitivity has also been done for the yy-configuration, i.e., the source- and the receiverantennas are still horizontal but oriented orthogonal to the survey line (Figure 5). Two changes compared to the $\mathrm{xx}-$ configuration are relevant: Firstly, for the yy-configuration the area of maximum sensitivity for the horizontal permittivity spreads to larger offsets, while the area of maximum sensitivity for the vertical permittivity is limited to small offsets. Secondly, this configuration is at small offsets $(<0.3 \mathrm{~m})$ sensitive to both, the horizontal and the vertical permittivity. This is in contrast to the $\mathrm{xx}$-configuration, which was not sensitive to the vertical permittivity at short offsets. Note also, that for both configurations, $\mathrm{xx}$ and $\mathrm{yy}$, the sensitivity for the horizontal permittivity is larger than for the vertical permittivity. This difference is more pronounced in the yy-configuration.
Also the sensitivity to the electric permittivity of the halfspace below the subsurface interface was computed (Figure $6)$. Note, that the direct field is not sensitive to the electric permittivity of the lower halfspace and, therefore, the sensitivity of the full field and the one of the reflections only are identical. Thus, only the full-field sensitivity is plotted in Figure 6. Because the recorded signal does not travel through the lower halfspace, the sensitivity to the electric permittivity of the lower halfspace is based completely on the reflection coefficient. Therefore, the amplitude of the sensitivity of the lower halfspace is one to two orders smaller than the amplitude of the sensitivity of the sandwiched layer. Otherwise, the sensitivity of the lower halfspace exhibits the same features with respect to the $\mathrm{xx}$ - and yy-configuration as for the sandwiched layer.

We computed the sensitivity as well for two situations with vertical antennas. These for GPR rather uncommon situations 

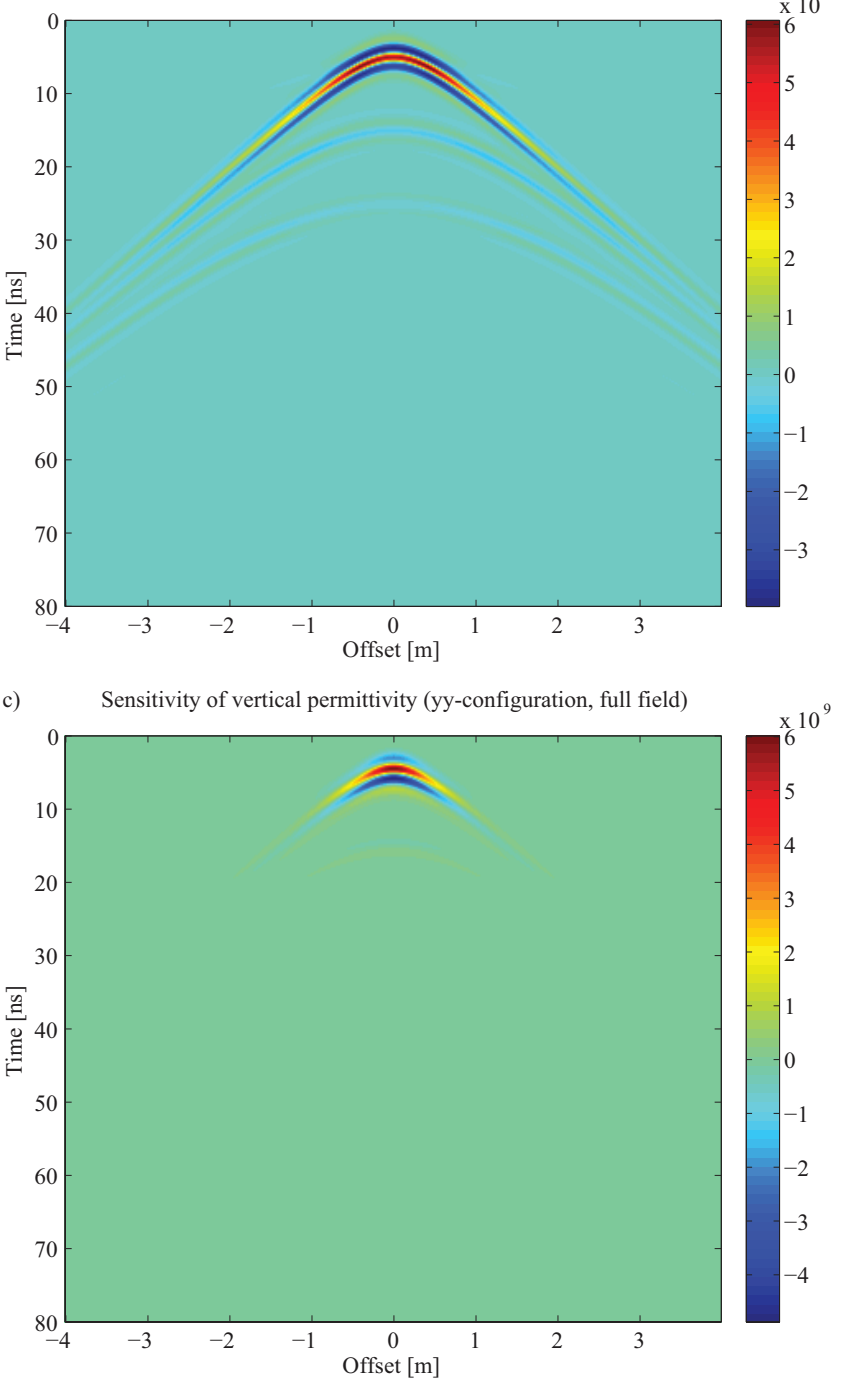

b) Sensitivity of horizontal permittivity (yy-configuration, reflections only)
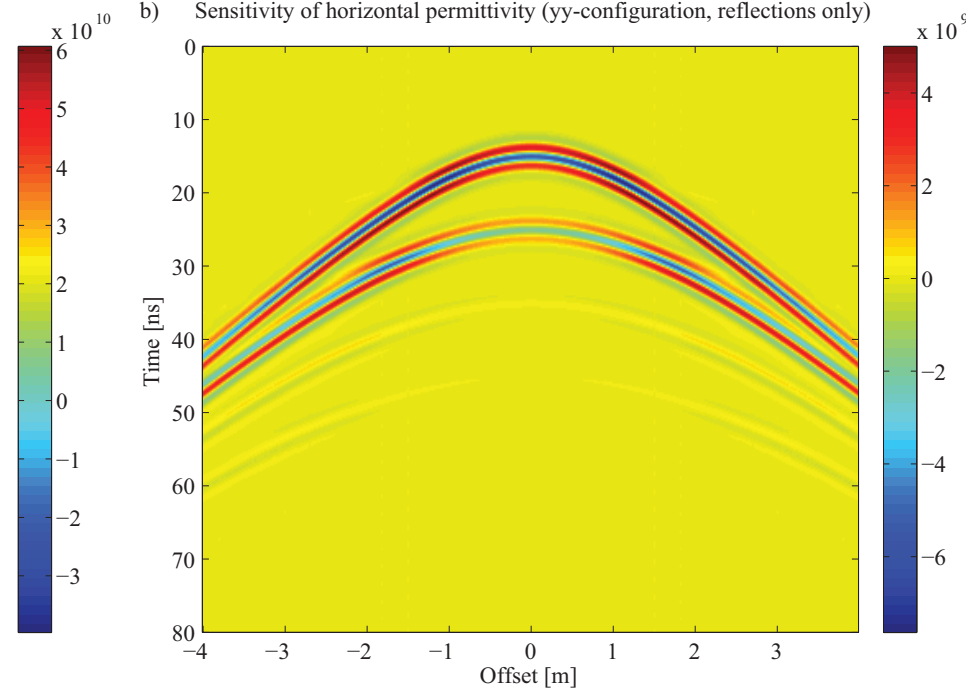

d)

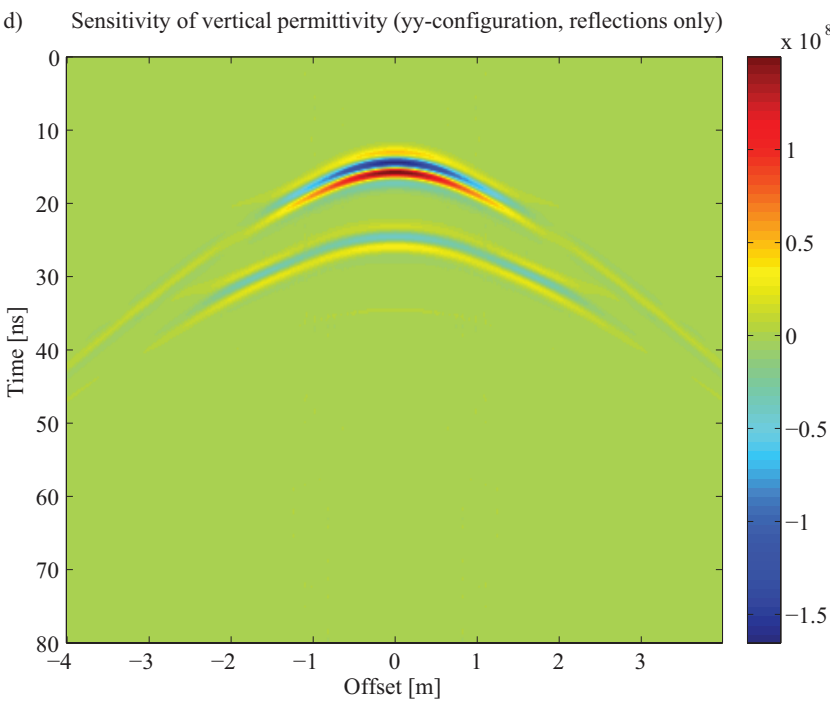

Fig. 5. Same as Figure 4, but for the source- and the receiver-antennas oriented orthogonal to the survey line (yy-configuration).

are, firstly, the zx-configuration, where one antenna is vertical while the second antenna is oriented horizontally and parallel to the inline direction, and, secondly, the zz-configuration, where both antennas are vertical. While the sensitivity to the electric permittivity of the first layer for the zX-configuration is similar to the $\mathrm{xx}^{-}$or yy-configuration, it is an order of magnitude smaller for the zz-configuration. Thus, there is no benefit in using one or two vertical antennas.

\section{CONCLUSIONS}

A fast analytical modeling code has been developed to model the electromagnetic fields in 3D in a stack of VTI-layers for low-frequency as well as for high-frequency applications. The code gives absolute freedom to the user with respect of source and receiver placement, orientation and type. If the source and the receivers are located at the same depth and at the same time at an interface, a small vertical separation needs to be introduced, because otherwise the wavenumber spectrum extends to infinity. The code is expected to be published as an open source package in 2014.

Having standard GPR acquisition with a small fixed antenna offset in mind, one might conclude, that the yy-configuration is best suited to image a VTI medium, because it is sensitive at small offsets to the horizontal as well as the vertical permittivity. However, two aspects should be taken into account: Firstly, for real data it might be necessary to also measure large offsets to estimate horizontal and vertical permittivity because an estimate based only on a single offset might not be reliable enough and, secondly, the sensitivity to the vertical permittivity is for the yy-configuration relatively smaller than for the xx-configuration. The sensitivity for the lower halfspace is much smaller than for the sandwiched layer, because the recorded signal does not travel through it. Configurations with vertical antennas do not show an increased sensitivity to the electric permittivity of the first layer and thus do not bring any benefit. 


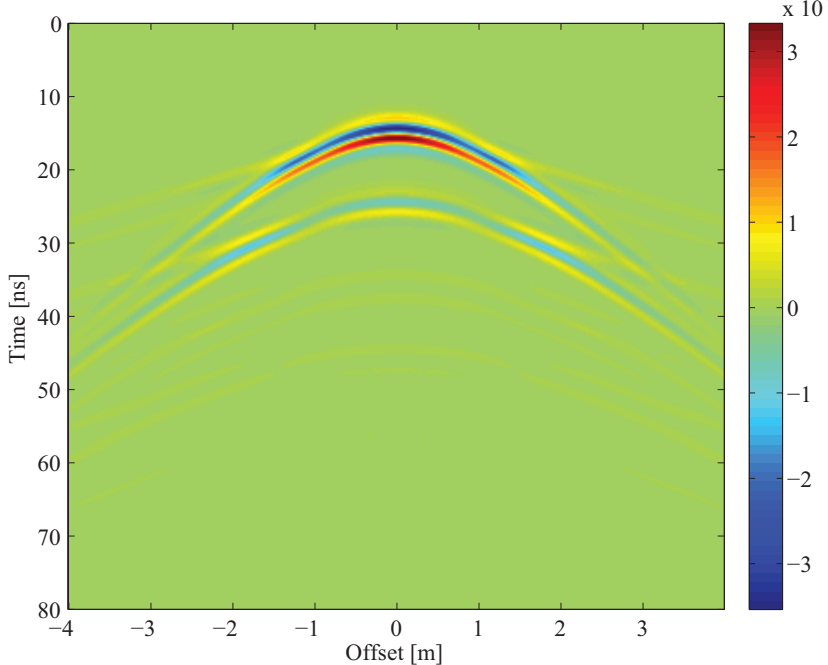

c) Sensitivity of horizontal permittivity (yy-configuration, full field)

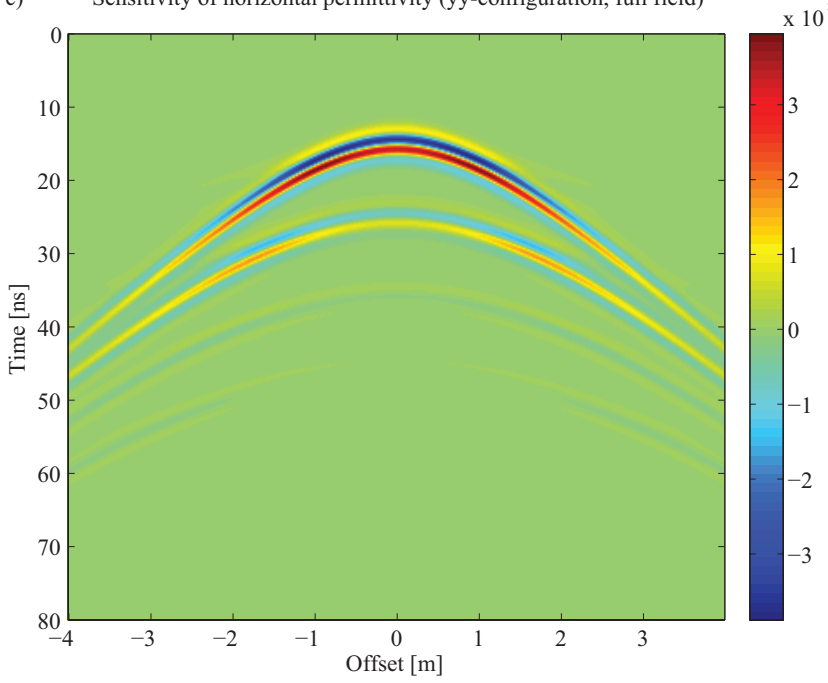

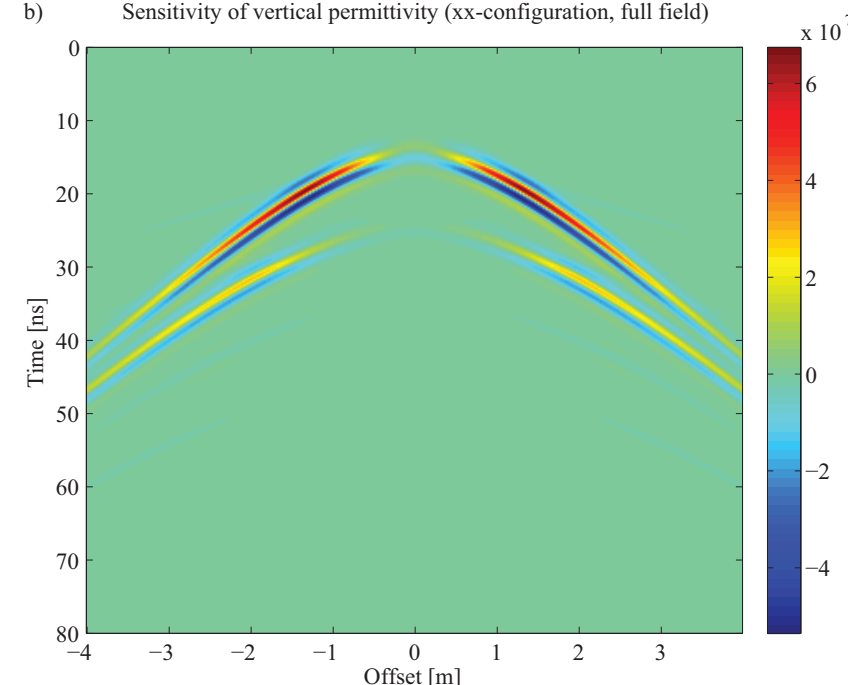

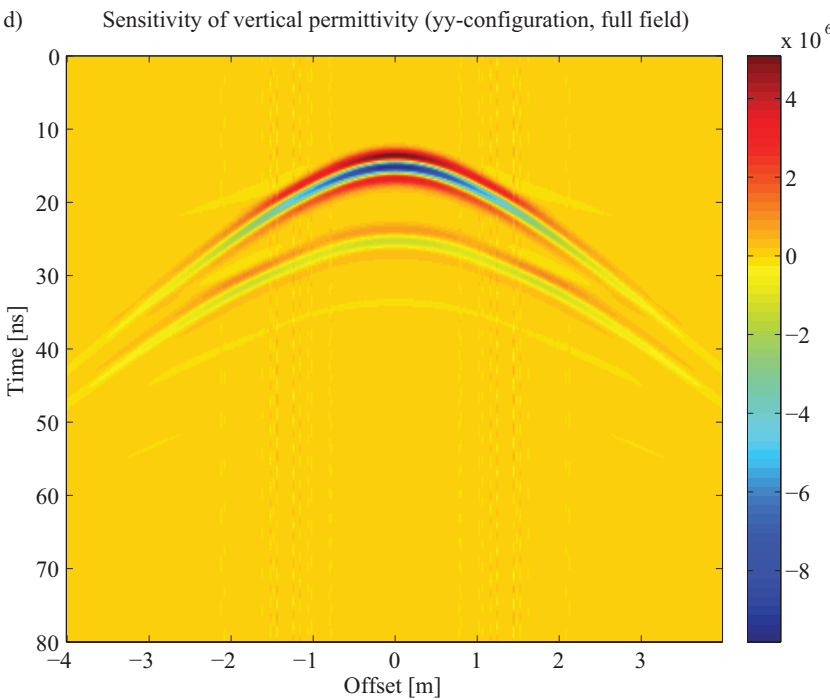

Fig. 6. Sensitivity to the lower halfspace: a) and b) show the sensitivity of the horizontal and vertical permittivity for xx-configuration, respectively, while c) and d) show the sensitivity of the horizontal and vertical permittivity for yy-configuration, respectively.

\section{ACKNOWLEDGMENT}

This research is supported by the The Netherlands Research Centre for Integrated Solid Earth Sciences (ISES).

\section{REFERENCES}

[1] J. Hunziker, J. Thorbecke, and E. Slob, "The electromagnetic response in a layered VTI medium: A new look at an old problem," Geophysics (under review).

[2] S. Constable, "Ten years of marine CSEM for hydrocarbon exploration," Geophysics, vol. 75, pp. 75A67-75A81, 2010.

[3] T. Eidesmo, S. Ellingsrud, L. M. MacGregor, S. Constable, M. C. Sinha, S. Johansen, F. N. Kong, and H. Westerdahl, "Sea bed logging(SBL), a new method for remote and direct identification of hydrocarbon filled layers in deepwater areas," First Break, vol. 20, pp. 144-152, 2002.

[4] R. Piessens, E. de Doncker-Kapenga, C. W. Überhuber, and D. K. Kahaner, Quadpack, A subroutine package for automatic integration. Springer-Verlag, 1983.

[5] J. H. Bradford, J. Nichols, J. T. Harper, and T. Meierbachtol, "Compressional and EM wave velocity anisotropy in a temperate glacier due to basal crevasses, and implications for water content estimation," Annals of Glaciology, vol. 54, no. 64, pp. 168-178, 2013.
[6] K. Matsuoka, T. Thorsteinsson, H. Bjornsson, and E. D. Waddington, "Anisotropic radio-wave scattering from englacial water regimes, Myrdalsjokull, Iceland," Journal of glaciology, vol. 53, no. 182, pp. 473-478, 2007.

[7] R. Martinez-Sala, I. Rodriguez-Abad, R. Diez Barra, and R. CapuzLladro, "Assessment of the dielectric anisotropy in timber using the nondestructive GPR technique," Construction and Building Materials, vol. 38, pp. 903-911, JAN 2013.

[8] D. Wang and G. McMechan, "Finite-difference modeling of borehole ground penetrating radar data," Journal of Applied Geophysics, vol. 49, no. 3, pp. 111-127, MAR 2002.

[9] J. Carcione and M. Schoenberg, "3-D ground-penetrating radar simulation and plane-wave theory in anisotropic media," Geophysics, vol. 65, no. 5, pp. 1527-1541, SEP-OCT 2000.

[10] P. Senechal, F. Hollender, and G. Bellefleur, "GPR velocity and attenuation tomography corrected of artifacts due to media anisotropy, borehole trajectory error and instrumental drifts," in GPR 2000: Proceedings of the Eighth International Conference on Ground Penetrating Radar, ser. Proceedings of the Society of Photo-Optical Instrumentation Engineers (SPIE), Noon, DA and Stickley, GF and Longstaff, D, Ed., vol. 4084, 2000, pp. 402-407. 